\title{
Philonsorbonne
}

5 | 2011

Année 2010-2011

\section{La psychologie et les structures sociales. À partir des recherches d'Olivier Revault d'Allonnes}

Frédéric FRUTEAU DE LACLOS

\section{OpenEdition}

1 Journals

Édition électronique

URL : https://journals.openedition.org/philonsorbonne/340

DOI : $10.4000 /$ philonsorbonne.340

ISSN : 2270-7336

Éditeur

Publications de la Sorbonne

Édition imprimée

Date de publication : 15 mai 2011

Pagination : 63-81

ISBN : 978-2-85944-680-2

ISSN : 1255-183X

Référence électronique

Frédéric FRUTEAU DE LACLOS, « La psychologie et les structures sociales. À partir des recherches d'Olivier Revault d'Allonnes », Philonsorbonne [En ligne], 5 | 2011, mis en ligne le 03 février 2013, consulté le 08 juin 2021. URL : http://journals.openedition.org/philonsorbonne/340 ; DOI : https:// doi.org/10.4000/philonsorbonne.340 


\title{
La psychologie et les structures sociales À partir des recherches d'Olivier Revault d'Allonnes
}

\author{
Frédéric Fruteau de Laclos \\ Maître de conférences \\ à l'université Paris1 Panthéon-Sorbonne
}

Je m'intéresse depuis la fin de ma thèse à une certaine forme de psychologie qui a pris naissance dans l'entre-deux-guerres. Cette psychologie a été baptisée par son inventeur, Ignace Meyerson, «psychologie historique, comparée, objective». Elle est devenue «anthropologie historique », à travers les travaux sur la Grèce de Jean-Pierre Vernant, son disciple le plus célèbre, tout en continuant de recevoir des prolongements originaux en psychologie de l'enfant, avec Philippe Malrieu, ou en philosophie de l'art, avec Olivier Revault D'Allonnes.

Dans les années d'après-guerre, cette psychologie se présentait comme compatible avec le marxisme : une authentique psychologie de la libération et du progrès, bien plus libératrice que ce que promettait au même moment le pavlovisme, seule psychologie officiellement tolérée par le Parti Communiste. «Une bonne psychologie historique suppose de bonnes études marxistes ", écrivaient ainsi Malrieu et Vernant dans un article rédigé à quatre mains ${ }^{1}$. Sans doute les auteurs espéraient-ils que le marxisme se tournerait vers cette «bonne» psychologie. Ce ne fut pas le cas. L'attaque vint d'un autre côté, bien moins réductionniste, bien plus philosophique, que celui auquel ils s'attendaient. Bientôt en effet, avec Louis Althusser et ses élèves, le marxisme, du moins en France, devint structuraliste, et ce structuralisme, profondément anti-psychologiste, l'emporta dans les esprits et les revues qui avaient été un moment tentés par l'aventure de Meyerson et des collaborateurs de son Journal de psychologie.

1. La Pensée, ${ }^{\circ}$ 61, mai-juin 1955, p. 124-136, repris dans J.- P. Vernant, Passé et présent : contributions à une psychologie historique, Roma, Ed. di storia e letteratura, 1995, p. 61-88. 
L'histoire, on le sait, ne s'arrête pas là, car à la pensée des structures, triomphante dans les années 1960 et 1970, a succédé une attention renouvelée à tout ce qu'elle laissait de côté. Par un mouvement de balancier historique, correspondant à un basculement indissolublement philosophique et politique, les penseurs des années 1980 revinrent à la psychologie, tant négligée et même raillée par les champions des structures ${ }^{2}$. Le structuralisme corrélait psychologie et individualité ; et surtout, supposait l'hétérogénéité de la prise en compte des structures et du travail mené par le psychologue : le psychologue s'illusionne s'il croit pouvoir accorder une initiative aux agents ; le travailleur n'est ainsi que le «porteur » de la force de travail, il est totalement défini par des déterminations socio-économiques extrinsèques, il est seulement le nœud de certaines relations structurelles entre forces économiques ${ }^{3}$. Mais le structuralisme, en durcissant la différence entre psyché et société, a irrémédiablement disjoint ce qui devrait apparaître comme les deux pôles d'une unique tension dialectique. La psychologie qui lui a succédé, par exemple la «psychologie politique » d'un Jon Elster, a achevé de consacrer cette scission, lorsqu'elle a repris la distinction en vue cette fois d'opposer avec force le libre choix rationnel des agents au «holisme méthodologique » de la sociologie française ${ }^{4}$.

Il nous faut revenir à la psychologie historique et à certains de ses développements originaux : cette psychologie rejeta la grille de lecture et le programme d'action (ou d'inaction!) proposés par le structuralisme, sans pour autant défendre, par anticipation, les thèses individualistes de la psychologie contemporaine.

Une précision s'impose, relative à la marche de l'exposé. Le travail s'articulera en deux temps, conformément à la méthode d' " anamnèse » que j'emprunte à la sociologie des sciences contemporaine pour l'appliquer à l'histoire de la philosophie. L'ambition ici est de faire de l'histoire pour faire de la philosophie ; il me semble en effet qu'on aura de grandes chances de refaire de la philosophie et d'intervenir dans le champ des pensées actuelles au terme d'une recherche purement historique sur les concepts produits avant nous ${ }^{5}$. Dans un premier moment historique, nous exhumerons des pensées enterrées, des possibles philosophiques qui, pour des raisons souvent non-philosophiques, n'ont pas donné tout le «rendement » théorique qu'on pouvait en espérer. Dans un second temps, proprement philosophique, nous dégagerons de cette investigation historique les concepts susceptibles d'intervenir dans la conjoncture théorique actuelle; à vrai dire, nous les

2. Cf. la critique féroce de Canguilhem de la psychologie dans «Qu'est-ce que la psychologie ?» (Etudes d'histoire et de philosophie des sciences, Paris, Vrin, 1968, p. 364381), et la réponse tout aussi féroce, dans le «moment »suivant de la pensée française, de Pascal Engel dans Psychologie et philosophie (Paris, Gallimard, Folio-Essais, 1996, Introduction).

3. E. Balibar, Lire le Capital, t. II, Paris, Maspéro, 1968, p. 149-151.

4. Cf. Le laboureur et ses enfants, Paris, Minuit, 1987, p. 15-16 et p. 50-52.

5. Je me permets de renvoyer à un texte méthodologique à paraître dans la revue Philosophie sous le titre : «Pour une histoire souterraine de la pensée française ». 
mettrons nous-mêmes au travail, en montrant pourquoi et en quoi ils sont susceptibles de jouer un grand rôle dans les controverses présentes en philosophie. Cela suppose une confrontation des philosophèmes ainsi « retrouvés » avec les théories qu'on nous propose aujourd'hui. Je ferai ainsi suivre les résultats de la recherche d'un certain nombre de propositions théoriques, éventuellement rapportées à des entreprises réflexives contemporaines. On verra alors, je l'espère, le bénéfice philosophique que nous aura permis d'obtenir le détour par l'histoire.

\section{Histoire et problèmes de la psychologie historique}

Qu'est-ce donc que la psychologie historique? Qu'attendait d'elle son fondateur, Ignace Meyerson? Qu'en firent après lui ses lecteurs et disciples?

Que la psychologie doive se faire historique signifie que la somme des catégories ou des fonctions au moyen desquelles les hommes se pensent et pensent le réel, soit leur outillage mental, diffère du tout au tout selon les époques. La mémoire, le travail, la personne, mais aussi bien l'espace, le temps, l'histoire, la cause ou l'objet, n'ont pas toujours eu le même sens ni la même portée pour les groupes humains qui ont employé de telles notions et qui ont réfléchi grâce à elles.

Plus précisément, Meyerson a de son propre aveu souhaité élaborer une «psychologie objective, comparative, historique». Soulignons immédiatement que son héritier spirituel le plus célèbre, l'helléniste JeanPierre Vernant, a réduit le projet aux dimensions d'une psychologie, et même d'une anthropologie, simplement historique, en s'employant à faire ressortir la grande originalité des Grecs dans la formulation et l'utilisation des fonctions psychologiques. Ce faisant, le disciple le plus fameux de Meyerson a restreint les potentialités offertes par la discipline. En se penchant sur les Grecs, il a eu tendance à repérer trop facilement le sens d'un progrès, malgré toute la finesse de ses analyses sur l'avènement de la raison en Grèce. Il s'est employé avec constance, et même avec insistance, à marquer la discontinuité entre mythe et raison, étayant à nouveaux frais la thèse d'un « miracle grec ». On pourrait souligner que cette vectorisation de l'histoire est présente chez Meyerson, qui n'a jamais renoncé à l'idée d'un progrès de la raison dans la conscience humaine. Mais à tout le moins, l'histoire, même soumise aux scansions d'un progrès, était le moyen, pour Meyerson, d'instaurer des comparaisons. Vernant a perdu de vue la visée comparative de la psychologie meyersonienne. Marcel Detienne a très récemment souligné que, chez Vernant, l'Homme grec en était petit à petit arrivé à porter le poids de l'Idéal humain qu'avait échoué à incarner l'Homme soviétique 6 . Parallèlement s'évanouissait l'esprit comparatiste,

6. Comparer l'incomparable, Paris, Le Seuil, 2009, Chap. VI et VII. 
l'Homme grec bénéficiant d'un traitement à part, exceptionnel, alors même qu'avaient commencé par être abordés transversalement les «problèmes de la guerre » et les «problèmes de la terre ». Des ouvrages collectifs consacrés par Vernant aux seuls Grecs ont paru sous ces titres, qui diffèrent du tout au tout de l'esprit résolument transdisciplinaire des volumes coordonnés quelques années auparavant par Meyerson, les Problèmes de la couleur et les Problèmes de la personne?.

Une dimension doit encore être soulignée: Meyerson jugeait que sa psychologie était objective. Quel sens cela a-t-il de demander à la psychologie d'être objective? La psychologie n'est-elle pas la science de l'intériorité, accessible seulement par introspection? La psychologie est objective, pour Meyerson, au sens, non pas où elle nie la subjectivité, loin s'en faut, mais au sens où elle prend pour objet l'objectivation de l'esprit, c'est-à-dire des sujets, dans les œuvres, œuvres de culture et de civilisation. Cela veut dire que les faits de civilisation ne sont pas seulement des choses : ce sont des signes ou des symboles à travers lesquels s'expriment les fonctions psychologiques. Il importe toutefois de marquer qu'on ne trouvera pas l'esprit hors des œuvres. Certes, par-delà Durkheim et toute la sociologie, on s'intéressera à la dimension mentale des faits sociaux. Mais contre Bergson et sa philosophie du moi profond se ressaisissant de l'intérieur par intuition, on affirmera l'extériorité, ou l'extériorisation, de l'esprit dans ses œuvres. On contestera pour finir à la psychologie de laboratoire la capacité d'appréhender les opérations de la pensée : ce n'est jamais 《à vide», mais toujours «en situation», à travers ses conduites, qu'on pourra comprendre les cheminements de l'esprit humain.

Enfin, et ce point n'est pas le moins important, l'entreprise meyersonienne s'ouvre sur une psychologie originale, c'est-à-dire sur une science singulière de l'individu dans ses rapports avec le collectif. Peut-être cette originalité se perçoit-elle mieux chez les élèves de Meyerson que chez le maître lui-même : soucieux surtout de marquer la spécificité du mental face aux institutions sociales, face à la pureté de l'esprit des philosophes, enfin face à la matérialité brute travaillée par la psycho-physiologie, Meyerson a peu insisté sur l'individualité et les relations interindividuelles. Sans doute y voyait-il aussi le risque d'une psychologie sociale libérale pour laquelle il avait peu de goût. Dans tous les cas, il revenait à certains disciples d'aller plus loin que lui dans ce domaine. Il est vrai qu'ils y étaient portés par leur objet d'études respectif. Olivier Revault d'Allonnes, attentif à l'évolution de la fonction de création dans les arts, était amené à se pencher tout particulièrement sur la génialité supposée des grands artistes. Philippe Malrieu, venu de la psychologie de l'enfant, développait et prolongeait la psychologie historique en une puissante «psychologie sociale

7. Cf. les ouvrages récemment édités par Detienne, où se retrouve l'esprit comparatiste de Meyerson, Tracés de fondation, Louvain-Paris, Peeters, 1990, Transcrire les mythologies. Tradition, écriture, historicité, Paris, A. Michel, 1994, ou encore Qui veut prendre la parole?, Paris, Le Seuil, 2003. 
génétique $^{8} »$. Il m'a paru naturel, dans le cadre d'une publication de l'Université Paris-I, de me concentrer sur le travail du premier, qui fut longtemps professeur de philosophie en ces mêmes lieux.

\section{La philosophie de l'art d'Olivier Revault d'Allonnes}

On pourrait s'étonner de ce que nous parlions de Revault d'Allonnes comme d'un disciple de Meyerson. N'est-il pas connu surtout pour être l'un des introducteurs en France de la pensée de l'Ecole de Francfort, notamment d'Adorno (ce qu'il revendique lui-même'), tout en étant l'un des chefs de file de l'esthétique française, à travers la direction, assurée avec Etienne Souriau et Mikel Dufrenne, de la Revue d'esthétique? C'est que, tout en faisant connaître la Théorie Critique, et parallèlement à sa collaboration avec Dufrenne, il a été à l'école de Meyerson, et cela littéralement: mis par Souriau sur la piste du séminaire professé par Meyerson à l'Ecole Pratique des Hautes Etudes, il l'a assidûment fréquenté et y est même intervenu à trois reprises alors qu'il préparait sa thèse ${ }^{10}$.

Disons un mot de ce que Revault entend retenir de Meyerson, ce qui l'a frappé dans son enseignement et sa lecture et qu'il a mis à profit dans sa propre recherche : la notion de forme des œuvres. L'histoire de l'art ne se réduit pas à des faits, même mis en séries, mais à des réseaux d'œuvres, renvoyant elles-mêmes à un esprit au travail en vue de produire des formes structurées. Dans l'hommage tardif qu'il rend à Meyerson, Revault retrace lui-même l'histoire complexe de ce concept de «forme », depuis la première réception des théories de la Gestalt, jusqu'à l'emploi technique qu'en fait son maître dans la lignée par exemple d'un Focillon, et cela avant même que n'éclose le paradigme structuraliste. Mais Meyerson n'a pas seulement fourni à Revault le concept adéquat pour penser son objet, les formes ou les structures des œuvres. Tout comme Meyerson, il fait travailler, sous les structures, une notion centrale de la psychologie historique, celle de «fonction psychologique». La psychologie historique ajoute la considération de l'histoire des œuvres, des changements de structures, qu'elle fait dépendre de l'évolution des fonctions. Les structures, il faut les croiser, mieux les traverser. On doit développer son travail transversalement à elles, et aller plus loin qu'elles. Revault est intéressé, plus que par les

8. La construction de l'imaginaire, Bruxelles, C. Dessart, 1967, rééd. sous le titre: La construction des imaginaires, Paris, L'Harmattan, 1999, p. 49.

9. Cf. notamment «Adorno non adorno », Revue d'esthétique, 1975, n 1, p. 171-191 ; «La raison en question. Premier débat autour de l'"Ecole de Francfort" ", avec Roger Dadoun, Gerhard Höhn et Marc Jimenez, Raison présente, n 36, octobre-décembre 1975, p. 41-55; «Autoportrait alphabétique », Revue d'esthétique, n³8, février 2001, p. 251-253.

10. Cf. son témoignage, «La psychologie historique et l'art», et les titres de ses exposés, dans F. Parot, Pour une psychologie historique. Ecrits en hommage à Ignace Meyerson, Paris, PUF, 1995, respectivement p. 131-135 et p. 111-114. 
structures artistiques prises en synchronie, par le passage d'un type de structure à un autre, selon une approche diachronique attentive aux transformations de l'outillage mental des groupes humains. Il y a là un point de convergence que Revault se reconnaît avec son camarade de la Sorbonne Gilles Deleuze. Pas plus que Deleuze, Revault n'a été structuraliste; il a toujours vu au-delà du structuralisme. Mais Deleuze l'a fait parce qu'il était bergsonien, alors que Revault est meyersonien : «trop historien» pour $s$ 'arrêter à la seule considération des formes ou des structures ${ }^{11}$.

Meyerson est bien un de ceux qui affirment avec force qu'on ne trouvera pas de structures sans fonctions psychologiques, et sans histoire ou évolution des fonctions. Mais le problème de la psychologie historique, telle qu'elle a été effectivement pratiquée par son fondateur, tient à l'abstraction de son abord des fonctions. J'ai traité ailleurs ${ }^{12}$ de l'abstraction des fonctions chez Meyerson. Qu'on y voie l'influence du «spiritualisme » de Brunschvicg, de Cassirer (comme je l'ai dit alors dans ces pages), ou de la prégnance chez Meyerson de modèles hérités de l'école sociologique française, via Mauss et Gernet (comme on pourrait être tenté de le penser en songeant à l' « hyperspiritualisme » de Durkheim), ne change rien au constat suivant : il faut préciser le sens, aussi bien la signification que la situation, des fonctions psychologiques. Revault est un de ceux qui s'y emploient le plus activement. Rappelons d'un mot en quoi consiste l'abstraction en question : distinct à la fois du social et du physiologique, le mental consiste pour Meyerson en une réalité planant au-dessus des individus en lesquels il s'incarne ou se réalise. Or, il faut aller voir très concrètement ce que les individus font des fonctions qu'ils reçoivent ou perçoivent, comment ils modifient l'héritage psychologique-collectif, et contribuent à le façonner singulièrement, en une dialectique de l'intériorisation et de l'extériorisation de données structurées (que la structuration soit biologique, sociale ou symbolique). Sur tous ces points, le travail de Revault d'Allonnes est décisif.

Je voudrais insister sur ce qui pourrait passer, si l'on n'y prenait pas garde, pour des incohérences voire des contradictions, et qui est en réalité le signe d'une très grande subtilité de Revault, sa capacité à naviguer entre des inconciliables, et à dialectiser des opposés. Si nous devons relever sa prise en compte de l'individu, ou des individus pourvu qu'ils soient saisis en situation, par-delà les prescriptions de son maître Meyerson, il nous faut également être attentif au maintien simultané de son exigence, opposée en ce cas à l'individualisme, de la considération des structures : l'individu ou la

11. Cf. la remarque livrée à François Dosse lors d'un entretien en préparation de la biographie croisée de Deleuze et Guattari : «Il [Deleuze] n'est pas plus entré dedans [dans le structuralisme] que moi. Qu'il y ait des structures, c'est évident, mais elles sont soumises à l'histoire, elles évoluent, sur ce plan je suis un élève de Meyerson. À ce titre, il n'y a pas de structures à la limite mais des processus ».

12. Dans «Euvre, fonction et société dans la "psychologie historique" d'Ignace Meyerson », Revue d'histoire des sciences humaines, $\mathrm{n}^{\circ}$ 17, Décembre 2007, p. 119-136, et «Vernant et Meyerson. Le mental, le social et le structural », Cahiers philosophiques, Dossier «Vernant, philosophe », $\mathrm{N}^{\circ} 112$ / décembre 2007, p. 9-25. 
subjectivité doivent être considérés sur fond des déterminismes qui structurent l'entour ou l'environnement.

\section{« Connaître les structures pour les changer »}

Il semble aller de soi que le critique d'art ou le philosophe de l'art ne puisse faire l'économie de la subjectivité de l'artiste pour rendre compte de la forme des cuvres : on ne cesse d'y insister sur la singularité à nulle autre pareille du génie créateur et sur celle de ses œuvres. Revault se méfie toutefois de cette référence au «sujet», source d'une libre et géniale créativité.

On le voit à sa réaction lors d'un débat auquel il participe et qui oppose en 1968 structuralistes et humanistes. Alors que Jean-Pierre Vernant vient d'interpréter son intervention comme un éloge de la décision humaine en art, il s'écrie : «Ce n'est pas une décision. C'est une opération élémentaire. Je ne veux pas de votre décision. Cela sent la psychologie à plein nez, votre

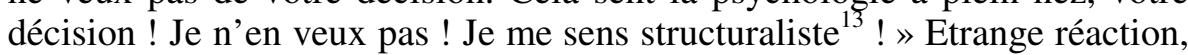
au premier abord, de la part d'un penseur qui vient d'affirmer : «les artistes doivent connaître les structures, mais pour les changer ${ }^{14} »$. Une psychologie de l'art, voire une pensée de l'individu-artiste, ne doivent-elles pas, dans ces conditions, prendre le relais d'une philosophie des structures ?

Cette étrangeté n'est qu'apparente, et elle s'explique parfaitement : ce n'est pas toute psychologie qui est rejetée par Revault, c'est la psychologie individualiste, celle qui triomphait avec le spiritualisme français et qui fait un retour en force aujourd'hui, sous des formes scientifiques différentes quoique politiquement homogènes (réactionnaires). Or, Revault se veut marxiste, et s'il est psychologue, c'est sur un mode inconciliable avec cet individualisme, d'une manière qui prend en considération le tout des structures matérielles et sociales en lesquelles s'ancre le travail de l'art. La position est difficile à tenir, entre la singularité de la création artistique, à laquelle Revault tiendra aussi jusqu'au bout, et même à la liberté attachée à cette création, et la détermination générale, universelle, des structures, dans les institutions de l'art comme en dehors de l'art. Elle est assumée de façon éclatante dans la thèse que Revault va soutenir peu après 1968, et dont le titre évocateur est La création artistique et les promesses de la liberté. Le texte se présente comme une suite de monographies; la Conclusion, un inédit en forme de synthèse, essaie de dégager des perspectives générales ${ }^{15}$.

Revault renvoie dos à dos les deux positions antagonistes de la création ex nihilo défendue par la philosophie individualiste de l'art et de la détermination intégrale de l'invention par les structures selon le point de vue marxiste ou sociologique. Il ne peut s'agir de création ex nihilo, car aucune

13. Structuralisme et marxisme, Paris, UGE, 10/18, 1970, p. 312.

14. Ibid., p. 288.

15. Esthétique et marxisme, Paris, UGE, 10/18, 1974. 
pensée ni aucune œuvre ne se bâtit à partir de rien, aucun esprit ne s'exerce à vide ; toute création suppose au contraire une foule de matériaux puisés dans la situation historico-sociale. Pour autant, aucune œuvre ne se réduit non plus au simple assemblage de «données » trouvées dans la situation.

Plaçons-nous au cœur de la situation, au moment même de la création ce que fait Revault à huit ou neuf reprises dans sa Thèse, qui se présente, je le rappelle, comme une rhapsodie totalement revendiquée. On ne comprendra les artistes qu'à se placer avec eux à ras de la création, dans le moment psycho-social où l'œuvre éclôt. Que voit-on alors ? L'artiste est celui qui fait fonctionner le champ à rebours ou à l'envers, qui renverse l'ordre des choses, qui intervertit et subvertit, fait dérailler et travailler dans une direction inhabituelle, les matériaux disponibles dans la situation. Il s'agit de "nommer création le point où l'ensemble des déterminations qui pèsent sur l'artiste se met à fonctionner "en sens contraire" » : «Le propre de l'acte créateur est de faire fonctionner ces contraintes en un sens qui ne faisait pas partie des virtualités impliquées dans le système. La création n'est donc nullement l'apparition de l'indéterminé, mais celle de l'imprévisible, celle de l'autrement déterminé ${ }^{16} »$.

La situation elle-même comprend, dans les interstices entre les institutions, dans les failles de leur fonctionnement «normal », à titre de potentialités, les réalisations artistiques futures. Le «sujet» était appelé à créer plutôt qu'il n'a créé ex nihilo. Encore fallait-il, il est vrai, qu'un sujet se présente pour répondre à l'appel. Soit un exemple d'histoire de la musique développé par Revault: le cas de la gamme, de l'échelle des sons utilisés. $\mathrm{Au} \mathrm{XVI}{ }^{\mathrm{e}}$ siècle, Zarlino élabore le tempérament ancien, qui sera remplacé à la fin du XVII ${ }^{\mathrm{e}}$ siècle par le système de Werckmeister. Dira-t-on que l'on passe d'un système à l'autre comme d'une structure cohérente et fixe à une autre structure cohérente et fixe ? Ajoutera-t-on que l'on abandonne le paradigme ancien du fait de ses lacunes et de son incomplétude, qui appelaient en quelque sorte le nouveau paradigme ? Et qu'éventuellement, un bon musicologue serait capable de préciser les « règles de transformation permettant de passer d'un système à un autre système, telles que l'observation concrète $[\ldots]$ peut les saisir »? Un tel musicologue serait structuraliste: la portion de phrase qui vient d'être rapportée est tirée d'un entretien dans lequel Lévi-Strauss fournissait une définition du structuralisme. Se faire une telle idée du passage d'une structure à une autre, c'est, estime Revault, faire preuve d'une «théorie de l'histoire singulièrement naïve et idéaliste ${ }^{17}$ ». Il n'existe pas de «calendrier idéal » par rapport auquel Zarlino est «en retard », Werckmeister « à l'heure » et Bach et plus tard Schönberg «en avance». Il n'y a pas non plus moyen d'appliquer à l'histoire de la musique la définition du structuralisme : les règles dont parle Lévi-Strauss n'existaient nulle part avant la transformation

16. Ibid., p. 15-16.

17. Structuralisme et marxisme, op. cit., p. 286, de même que toutes les citations qui suivent dans ce paragraphe. 
effective, constatable dans le détail empirique et concret de l'histoire ! Ce qu'il faut relever, en historien, c'est qu'on a affaire dans chaque cas à des « replâtrages », «bricolages », « arrangements » plus ou moins bons des systèmes. D'un côté, tout système est cohérent, saturé, stable - la preuve, il est fécond (c'est le cas du système de Zarlino). «Et puis - je cite ici Revault-, les points faibles d'un système, plus ou moins obscurément connus et signalés, deviennent des déchirures.» Sur ces entrefaites, surgissent Werckmeister puis Bach, qui sont eux-mêmes «rigoureusement imprévisibles, littéralement impensables ».

Le structuralisme est certainement légitime en son ordre - qui est l'analyse des paradigmes, la structure formelle des œuvres d'art; particulièrement bien venu pour les systèmes musicaux. Revault y insiste à deux reprises : "Je pense avec François Châtelet que ce salut [des sciences humaines] doit s'opérer à l'aide du concept de structure $^{18} \gg$; et plus clairement encore : « les esthéticiens sont tenus à une exigence élémentaire, qui est de penser les systèmes sous peine de sombrer dans la gratuité ». Mais il ajoute qu'ils sont tenus aussi à «une exigence supérieure qui est de s'intéresser aux failles des systèmes, et plus aux failles qu'aux systèmes, sous peine de manquer totalement leur objet, qui est une œuvre d'art, donc une nouveauté, un éclatement du système, voire un antisystème délibéré » : «Les artistes doivent connaître les structures, mais pour les changer ${ }^{19}$ ».

On n'ira surtout pas croire que la structure à elle seule, le jeu dans la structure, suffise à rendre compte des changements. Ce serait là retrouver cet idéalisme que les structuralistes se donnent pour toute théorie de l'histoire : l'espace des structures serait riche de potentialités, il suffirait d'y puiser pour «créer». Revault se fait une conception à la fois plus politique et plus empirique de l'invention dans les paradigmes. Les structures, sociales mais aussi esthétiques, la tradition de l'art que l'on pratique - tout cela est répressif ou oppressif. Contrairement à ce qu'affirme sur ce point l'École de Francfort, notamment Herbert Marcuse, l'histoire de l'art agit comme une force d'empêchement, l'ordre esthétique est un ordre répressif : «les forces de la tradition, de la continuation ont une fonction précise d'étouffement et de répression ${ }^{20} »$. Cette emprise est telle qu'elle ne peut manquer de susciter la révolte ou le soulèvement : la création est le résultat de cette réaction, la création est cette réaction même :

Les artistes ne créent une œuvre nouvelle que lorsqu'ils y sont obligés par l'usure, le coincement, les contradictions de leur art. Les hommes créeront une société nouvelle le jour où ils n'auront pas d'autre solution.

S'il est vrai qu'on ne crée que lorsqu'on est condamné à créer ; s'il est vrai que c'est l'accumulation des contraintes qui force la main de la création, alors il n'y a pas à regarder avec fatalité et résignation la montée des forces

18. Ibid., p. 284

19. Ibid., p. 288.

20. Esthétique et marxisme, op. cit., p. 18. 
sociales de détermination. N'oublions jamais que c'est dans l'exacte mesure où cette société-ci ne peut s'empêcher de contraindre qu'elle débouche par ses processus internes sur une autre société que nous ne pouvons nous empêcher de créer ${ }^{21}$.

On invente toujours pour lutter, pour contrer la répression dans le champ. Si «creux» il y a, c'est celui, empirique et terre à terre, des conditions d'existence viles ou misérables auxquelles nous conduit parfois la domination, et certainement pas le réservoir des virtualités de transformations formelles chères aux structuralistes.

On comprend que les protagonistes du débat de 1968 aient eu du mal à situer Revault: il en passe par les structures pour atteindre aux hommes concrets, en situation. Pour autant, est-il individualiste ? Fait-il dépendre des hommes, de leurs décisions conscientes, le renversement des paradigmes ? Approfondissons maintenant ce point, car on serait tenté de penser que les révolutions en art sont suspendues à l'apparition contingente d'un génie, puis au libre déploiement de son inventivité. Il est nécessaire en effet que quelque force agisse, susceptible de faire basculer le déterminisme en liberté, de faire devenir ou advenir la liberté à partir du déterminisme et en dépit de lui, selon une formule lâchée par Revault en 1973. Nous devons parler, dit-il alors, « de la création comme point où les déterminations de tous ordres deviennent autre chose, se mettent à fonctionner dans l'autre sens. Non pas déterminisme ou liberté, mais déterminisme devenu libertét ${ }^{22} »$. Ce « devenir » pourrait receler bien des problèmes : ce mouvement ou cet élan doit avoir une cause, la question étant de savoir en quel sens cette cause peut être cause de soi. Nous allons voir que, si ce n'est pas un sujet libre, maître de lui-même et dominant le simple enchaînement des causes et des effets, c'est un sujet vivant, une vie luttant pour ne pas être étouffée.

L'affaire est ainsi plus compliquée, et c'est là un effet de la complexification par Revault des principes de la psychologie historique. Des thèses subtiles et balancées, «dialectiques », doivent être défendues contre les doctrines unilatéralement opposées de la détermination exclusive par les structures et de l'indépendance absolue de la création. L'histoire de la «fonction de créativité », appelée par Revault «esprit de création », est mélangée, impure et bigarrée, à l'image du rapport entre les principes qui la conditionnent. Plutôt que la décision délibérée d'innover prise par un sujet, conscient de lui-même et du monde, nous devons parler de la résistance d'une vie qu'on cherche à écraser, la réaction d'un vivant qu'on opprime. Au terme d'un long détour, et sous l'influence conjuguée d'Adorno et de Dufrenne, Revault dégage la voie d'une esthétique de la vie qui évite la psychologie individualiste, contourne l'idéologie de la théorie de l'agir rationnel, tout en rendant compte de l'invention sous les structures, entre les interstices ou dans les failles de leur systématicité. 


\section{La vie est sujet}

Cette découverte d'une voie originale est exposée dans un long texte écrit en hommage à Dufrenne, "La désublimation artistique ». Il sera repris dans la «monographie » Plaisir à Beethoven ${ }^{23}$. Arrêtons-nous un instant à ces pages.

Dans son abord de l'artiste, Revault d'Allonnes entend éviter la métaphysique autant que le positivisme. Il faut s'opposer au positivisme qui, en s'attachant à la structure formelle des œuvres, se dispense de rendre compte du travail humain dont ces structures sont les effets. Les faits picturaux, musicaux, en général artistiques, ne sont pas des choses. Mais contre la métaphysique - et Bergson est l'un des meilleurs exemples, en tout cas des plus récents, d'une psychologie métaphysique -, l'enjeu est de dénier l'absolu d'un sujet placé en retrait de l'expérience, hors d'elle, cause de soi qui ne serait effet d'aucune autre cause, conception métaphysique de la liberté qui fait le fond de l'Essai. Ainsi Bergson et Durkheim sont-ils renvoyés dos à dos ${ }^{24}$. Aussi bien s'agit-il de naviguer entre eux. On ne doit ni hypostasier le travail en sujet ni réifier les œuvres et les convertir en simples objets. Cependant, entre ces deux termes extrêmes, n'existe-t-il pas la tension, malgré tout, d'une polarité ?

Ce qu'il faut voir, c'est l'effet de la «loi du profit» sur un «quelque chose » que nous devons nommer « la vie». Car, dit Revault d'Allonnes, la loi du profit sépare le sujet de l'objet, la possibilité de la satisfaction artistique dans la création et l'œuvre elle-même, comme produit de cette activité. D'un côté, elle réifie l'œuvre, elle en fait une «belle œuvre», inaccessible dans le ciel des plus hauts achèvements de l'Humain; de l'autre, elle hypostasie le sujet, le fige dans la figure du génie artiste, sans égal, susceptible de devenir l'objet d'un culte pour les autres sujets. Ce qui est ainsi séparé, le sujet et l'objet étant littéralement inventés ou produits par cette séparation, c'est l'unité de la vie en sa valeur et sa teneur bifrons, à la fois activité (ou création) et œuvre.

Qu'il y ait là la vie, en sa dynamique propre, en même temps qu'en sa réalité anté-«humaine», présubjective, aussi bien que non-objective, c'est ce que prouve, à mon sens, la référence finale au «merveilleux » petit livre d'Etienne Souriau sur Le sens artistique des animaux ${ }^{25}$. Souriau entendait manifester dans cet ouvrage illustré son attachement, non à l'anthropomorphisme, mais au zoomorphisme; loin de vouloir montrer que l'humain est préformé dans l'animal, que l'animal est déjà humain, il visait à

23. Vers une esthétique sans entrave, Mélanges Mikel Dufrenne, Paris, U.G.E., 10/18, 1975, p. 155-196, repris dans Plaisir à Beethoven, Paris, Christian Bourgois, 1982, p. 107-176.

24. Ce sont là les deux auteurs du programme de l'oral d'agrégation de Revault, Cf. le témoignage confié à F. Dosse; ainsi que l' «Autoportrait alphabétique» de la Revue d'esthétique, op. cit.

25. Plaisir à Beethoven, op. cit., p. 237. Cf. également l'hommage rendu à Souriau, «L'enseignement de l'Esthétique », dans «L'art instaurateur », Revue d'esthétique, 1980, $\mathrm{n}^{\circ} 3-4$, U.G.E., 10/18, p. 224. 
ancrer l'humain dans l'animal, à souligner la présence du beau au cœur du vivant, à même toute vie ${ }^{26}$. À cette référence, il faudrait ajouter chez Revault tous ces indices que sont les guillemets utilisés dès qu'il parle du « sujet » ou de la «conscience», autant de termes relatifs, non pas originaires, mais dérivés de l'opération du Capital.

Nous pourrions nous demander pourquoi le Capital agit ainsi, pourquoi telle est sa loi ou son opération. Et cependant, la réponse va de soi : en coupant l' «objet» de son «sujet», il sépare la vie de son dynamisme, il ampute la créativité de sa visée, il coupe l'œuvre de ses conditions concrètes, vitales, d'apparition ou de création, et par là se donne les moyens, après avoir dénaturé l'art et l'artiste, de soumettre les autres hommes : l'œuvre, promue au rang de chef-d'œuvre, et l'artiste au rang de génie, apparaissent comme inatteignables, inimitables; les simples «mortels » qui auraient été tentés de s'exprimer et de s'épanouir en créant, en un mouvement de subversion de tous les ordres imposés d'en haut, en seront empêchés, par inhibition ou castration, par intimidation et écrasement.

Aussi bien, explique Revault - si des fois l'on avait un doute l' « objet » créé par l'artiste est un « anti-objet », sa vitalité est le signe d'une résistance à toutes les sédimentations ; tandis que le «sujet» est un «nonsujet », il n'existe pas séparé, ni de ses conditions matérielles, concrètes et parfois très basses, de son existence, homme ou animal comparable en cela à ses congénères («tout homme est tout l'homme », dira Revault en reprenant en forme d'hommage la formule de Sartre), ni de son travail de libération, de « désublimation artistique ». Qu'il y ait, qu'il doive y avoir, « désublimation artistique », selon la belle expression de Revault, signifie qu'on n'ira pas chercher dans l'art, ou chez l'artiste, les moyens d'un dépassement, d'une sublimation, de la condition qui nous est faite. L'artiste ne rachète pas, il ne sauve rien. Penser que l'art sublime, c'est estimer qu'il tend à contrebalancer la bassesse de la vie qu'on nous impose, par les produits les plus subtils et éthérés de la haute culture. Ce n'est certainement pas le cas : l'art ramène à cette condition ou à ces situations plus qu'il ne nous en détourne. Il désigne et dénonce le tort fait aux vivants. En un mouvement explicitement antifreudien, il faut en appeler à une saisie de l'art et du travail artistique comme mise au jour et refus de l'aliénation et de l'exploitation quotidiennes - toute une œuvre, par conséquent, de « désublimation artistique ».

Les deux pôles de la vie, les deux faces de l'existence, sont conçus comme créativité : il n'est pas question d'attendre d'avoir affaire au beau pour apercevoir des œuvres, mais nous appréhendons dans toute vie l'instauration d'une œuvre. Il est temps de dissocier l'art du beau, pour l'associer au désir, ou plutôt au plaisir, à la satisfaction de la libération. Comme dit Revault après Dufrenne, en dépit de certaine formule sur le désir dans les textes sur Adorno, il importe de lutter contre le désir comme manque, et de voir dans le plaisir un signe, mieux un critère, de toute

26. Cf. E. Souriau, Le sens artistique des animaux, Paris, Hachette, 1965, p. 7, 44-46. 
réalisation de soi et de son œuvre, du soi dans son œuvre, de l'œuvre portée par une vie.

Cette double face de la vie manifeste la continuité de l'attachement de Revault à la psychologie : toute la psychologie est là, dans cet effort pour cerner la créativité, pour la suivre en ses cheminements inventifs. Précisons bien : elle est tout entière là, et non en retrait, dans la localisation et la description de quelque retraite subjective, profonde et fondamentale, d'où sourdrait la créativité elle-même. La fonction de création n'a nul besoin d'un esprit en surplomb, de la hauteur toujours trop élevée du «génie». Elle se comprend, et ne se comprend que dans le corps à corps du vivant avec son travail.

Rappelons, au passage, les développements du Beethoven sur «la vie et l'œuvre »: oui, il faut prendre en considération l'homme Beethoven, sa « vie »; derrière les œuvres, il n'y a aucun mal à aller le chercher lui. Si on ne le fait pas, on se condamne à la froide impersonnalité de l'analyse des structures, objectives-objectales, du produit fini. Et l'on ne comprend rien à ce qui l'anime en réalité. Cette réalité n'est pas pour autant transcendante, ou réfugiée dans quelque insigne singularité du «grand homme»: une fois qu'on a saisi que l'œuvre se rattache à Beethoven créant, à l'esprit de créativité qui vivifiait et agitait l'esprit de l'homme Beethoven, on n'a pas besoin d'aller plus loin et de s'enfermer dans les menus faits de sa vie comme s'il s'agissait de pépites laissées derrière lui par une subjectivité incomparable et splendide, en un mot supérieure à la fois à tous les objets et à tous les sujets.

Sous cet aspect très «vitaliste », il faut reconnaître qu'il y a quelque côté bergsonien chez Revault. Mais un Bergson très particulier, ou très singulièrement interprété, non pas le Bergson «subjectiviste » de l'Essai celui-là a été rejeté, à travers le refus de sa «psychologie métaphysique », et il n'est question à aucun moment de finalement revenir à cette conception du sujet $^{27}$. Mais nous avons très certainement affaire à une conception de la vie : c'est bien la vie que Revault promeut à sa façon, en ses métamorphoses créatrices, en son évolution, irrésistible en dépit des obstacles que l'inertie lui oppose $\mathrm{e}^{28}$.

\section{Propositions pour une psychologie de la libération}

J'ai retracé l'histoire de la psychologie historique, en particulier le devenir d'un jeune chercheur trouvant au contact de Meyerson une voie dans

27. Cf. dans le volume d'hommage L'œuvre et le concept. Prétextes Olivier Revault d'Allonnes, Paris, Klincksieck, 1992, N. Tertulian, «La défense du sujet», p. 47-51, en particulier p. 49.

28. Cf. la référence à « une pensée des choses en train de se faire », dans l'interview ultime avec Dosse. 
laquelle s'engager. J'ai suivi les inflexions qu'il avait fait subir à ce «courant» de psychologie historique, du fait de son objet: il devait s'expliquer avec la thèse de la génialité supposée de l'individu artiste. Il découvre alors qu'il faut aller au-delà des structures, vers la créativité comprise comme esprit ou fonction de création; bien plus, qu'il faut voir cette fonction se déployer en situation - psycho-sociale -, saisir comment s'y prend un sujet pour inventer, malgré les obstacles qui lui sont opposés, que ce sujet soit un ou pluriel ${ }^{29}$. Nous avons été amené, en suivant le progrès de la pensée de Revault, à rendre compte de son approfondissement du sens de la subjectivité, en rapport avec les pensées d'Adorno et de Dufrenne : si le sujet créateur peut être un individu autant qu'un groupe, c'est que l'Humanité est mue par une force de vie infrapersonnelle, celle-là même qui se trouve investie, dans le meilleur des cas, dans des aventures de création individuelles ou collectives, entravée lorsque des forces de domination et d'exploitation séparent la créativité de ses créations, la vie de ses œuvres. Par là, Revault semble aussi éloigné de l'impérialisme intellectuel des structures que de la réduction de la politique aux seules raisons conscientes des individus.

Je l'ai dit, cependant : l'histoire de ce devenir est seulement le premier temps d'une démarche qui en comporte deux. Cette histoire n'a été retracée que pour permettre de renouer, en deçà des modes contemporaines ou des idéologies dominantes, avec certains philosophèmes qui ont été étouffés aussitôt que produits. Qu'ils aient disparu, ou aient paru ne pas pouvoir s'imposer, ne signale pas leur faiblesse conceptuelle, mais témoigne plutôt en faveur de leur puissance de changement, dans l'ordre des idées autant que dans le registre de l'action. Peut-être est-ce précisément parce qu'elles étaient vives que ces pensées ont été écartées, parce qu'elles demandaient des transformations qu'elles n'ont pas trouvé de relais dans la structure conservatrice du champ de la philosophie de leur temps. Dans les pages qui suivent, je m'efforcerai de fixer quelques acquis philosophiques de ce travail de pensée. J'avancerai des propositions «abstraites », enchaînement de notions tirées de l'histoire qui vient d'être racontée. Mais je tenterai dans le

29. Peut-être y aurait-il eu lieu d'insister plus tôt sur ce point, qui met à mal une interprétation purement «individualiste» de cette théorisation de la création artistique : le sujet n'est pas nécessairement singulier, il peut y avoir « génie » d'un peuple, ou d'une classe, comme il peut y avoir, malheureusement, aliénation de groupes entiers ; autrement dit, réussite aussi bien qu'échec de la créativité d'un collectif considéré comme sujet. Tels sont les deux exemples, centraux dans la thèse de 1973, du rébétiko grec d'une part, du design industriel d'autre part (La création artistique et les promesses de la liberté, Paris, Klincksieck, 1973, 2007, chap. VI et VII). Dans le premier cas, Revault analyse l'émergence et l'épanouissement d'un style musical des bas quartiers et de la pègre dans la Grèce du $\mathrm{XIX}^{\mathrm{e}}$ et de la première moitié du $\mathrm{XX}^{\mathrm{e}}$ siècle ; nul auteur en particulier ne se dégage, plutôt un faisceau de revendications obscures s'exprimant à travers paroles et musiques circulant en certains lieux, et en certains milieux. Dans le second cas, on a affaire à des productions en série qui prétendent au statut de «belles choses ». Revault montre que cette prétention est vaine : la «beauté » en question ploie toujours devant l'impératif de séduction de l'acheteur potentiel, elle demeure assujettie aux objectifs du rendement et de l'utilité. 
même temps aussi un second genre de contextualisation, par confrontation aux pensées présentes ou plus connues, qu'elles soient parentes ou opposées. Il s'agira ainsi, d'une part de dégager la valeur des concepts, sinon en soi, du moins au-delà de la situation qui les a vus naître, d'autre part d'engager cette valeur dans le moment présent, de l'affronter à ce qui se présente comme concepts de notre contemporanéité.

Partons de l'idée de structure. Toute structure est travaillée par deux sens de la liberté, conformément aux deux pôles qui la constituent. Pour avoir une structure, il faut et il suffit que l'on ait deux pôles pris dans l'interaction d'une relation hiérarchique ou de domination. Renvoyons ici à ce que Veyne appelle le « défi objectif » représenté par les « gouvernés ${ }^{30}$ », à ce qu'Edmond Goblot appelait déjà une «barrière et un niveau », et qui a donné chez Bourdieu la théorie de la « distinction ${ }^{31} »$. Deux pôles, dont l'un est en haut et l'autre en bas, dont l'un prétend être supérieur et exerce sa domination sur un ensemble d'inférieurs, instaure entre eux et lui une barrière, distinguant un niveau (d'aisance financière, d'éducation, capital économique ou capital symbolique), ou deux niveaux si l'on veut dont l'un est dit vulgaire, et l'autre élevé. Une structure est toujours hiérarchique ou hiérarchisante, dans la mesure même où la hiérarchie définit la structure.

Avant d'aller plus loin, disons un mot des références qui viennent d'être invoquées.

Le pain et le cirque de Paul Veyne a été «enrégimenté » par la pensée libérale : cette foisonnante «thèse », estime Jon Elster, ne démontrerait aucune thèse, du moins ne ferait fond sur aucune théorie du social, mais se contenterait de repérer, à travers un large parcours historique, une foule de «mécanismes de pensée » (librement) mobilisés par les individus ${ }^{32}$. Il me semble qu'il n'en est rien, mais que Veyne explicite au terme du parcours une loi générale du fonctionnement social, qu'il nomme le «pacte historique ». Cette loi lui permet de subsumer tous les petits mécanismes croisés dans la Grèce des Cités, la République romaine, enfin l'Empire romain : l'effort pour établir et maintenir la «relation entre gouvernés et gouvernants » est la raison des conduites disparates et en apparence si étranges, à nos yeux, des individus. L'existence d'un "pacte historique », dégagée dans Le pain et le cirque, signifie que certains individus - à vrai dire la plupart - acceptent d'être dominés, ils tolèrent la distance ou le surplomb des autres - en minorité -, du moment qu'ils y gagnent la sécurité de la stabilisation des relations sociales, et, de temps à autres, quelque

30. Paul Veyne, Comment on écrit l'histoire, «Foucault révolutionne l'histoire», Paris, Points-Seuil, 1996, p. 395, à propos de Le pain et le cirque, Paris, Points-Seuil, 1995 (cf. dans ce dernier texte p. 339-344).

31. Cf. Pierre Bourdieu, La distinction, Critique sociale du jugement, Paris, Minuit, 1979, et la récente réédition du livre d'Edmond Goblot, La barrière et le niveau, Paris, PUF, 2009.

32. Le premier chapitre de Psychologie politique (Paris, Minuit, 1990) est un commentaire de Le pain et le cirque. Cf. aussi d'Elster, Marx, une interprétation analytique, Paris, PUF, 1989, la section «Individualisme méthodologique », p. 19-23. 
compensation symbolique sous forme de jeux ou de pain. Autrement dit, tout ne dépend pas des individus et de leur libre volonté de se comporter ainsi plutôt qu'autrement. La structure sociale et la place qu'y occupent les dominants joue un rôle déterminant, même si, selon Veyne, tout ne se réduit pas à une causalité de type économique. En dépit de sa méfiance à l'égard de l'économisme, Veyne aboutit à une conception tout à fait conciliable avec la sociologie d'un Durkheim ${ }^{33}$. Rappelons que le sous-titre de son livre est «Sociologie historique d'un pluralisme politique». Il est vrai qu'il se préoccupe de savoir comment des individus réagissent face à la hiérarchie ou à la distance sociale. En ce sens, il élabore bien une "psychologie politique ». Pour autant, cette «psychologie politique»se révèle être une psychologie de la distance sociale, l'analyse de la réaction des individus confrontés à des structures sociales de domination.

D'autre part, dans sa Préface à la réédition de La barrière et le niveau, Bernard Lahire souligne l'importance de Goblot dans le projet, mené par Bourdieu et Passeron, d'une étude du mode de reproduction des élites via l'école et les grandes écoles. Elle ne fut jamais explicitée comme telle par Bourdieu, alors que Passeron a volontiers reconnu cette influence ${ }^{34}$. Elle correspond, explique Lahire, à une tentative pour dépasser les attendus des analyses marxistes, qui ne voyaient de distinction qu'économique: il s'agissait d'établir que les inégalités sont aussi culturelles, et que le capital des dominants est autant symbolique que financier. On prendra garde que ce dépassement du marxisme est interne à la tradition critique de la distinction inaugurée par Marx. Il n'est pas question pour les auteurs de disputer Marx comme s'il n'existait pas de domination, ainsi que le font les libéraux, mais bien d'étendre la théorie de la domination à un champ sous-estimé par Marx. Nous devons être plus radicalement critiques que Marx, en admettant que les mécanismes de soumission affectent la superstructure culturelle en plus de l'infrastructure économique. On s'explique, dans ces conditions, que Goblot ait pu jouer un rôle décisif dans l'évolution intellectuelle de Bourdieu et Passeron. Pour être bourgeois, et en particulier farouchement opposé à l'économisme marxiste ${ }^{35}$, Goblot n'en était pas moins attentif aux causes et aux effets culturels de domination. Loin d'être simplement «libéral », il s'efforce de toujours rendre raison des situations sociales de tension symbolique en lesquelles se trouvent plongés les individus.

Ces précisions faites, reprenons l'exposition de nos propositions pour une psychologie de la libération. Au sein de la structure, deux sens de la liberté apparaissent, conformément aux deux pôles dégagés. D'un côté,

33. Elster est conscient de cette proximité, mais il la déplore. Il reproche à Veyne, tout comme à Durkheim ou à Bourdieu, leur «fonctionnalisme», soit leur tendance à faire des réactions des individus les effets du fonctionnement de la structure sociale, de faire de la conduite des agents des fonctions du tout (ibid., p. 57-58).

34. Cf. l'entretien avec Raymonde Moulin et Paul Veyne, dont B. Lahire cite une version longue inédite dans sa Préface à La barrière et le niveau, op. cit., n. 2 p. X.

35. Cf. sur ce point sa Logique des jugements de valeur, Paris, A. Colin, 1927, «Exercice V. Théorie de la valeur de Karl Marx », p. 137-154. 
on trouve cette liberté formelle dont la psychologie individualiste méthodologique prétend qu'elle est la réalité sociale dernière, la seule sur laquelle pourraient s'appuyer les études des sciences humaines. À cette conception, il faut objecter, comme le faisait le structuralisme marxiste, que cette liberté est production idéologique. On aurait en effet tort de l'étendre à tous les membres ou à toutes les parties du corps social : le bourgeois n'est libre, ne peut se sentir libre, que parce qu'il a les moyens économiques de cette liberté, et, par ces moyens, la possibilité de se ménager du temps pour penser aux conditions de réalisation de sa liberté, non seulement de préservation de son corps et de sa vie, mais aussi de la réussite de son existence suprasensible - de son bien-être temporel autant que du salut de son âme.

D'un autre côté, nous devons être attentifs à un tout autre sens de la liberté (que le structuralisme ne prend pas en compte). La liberté en question n'est pas donnée, elle ne se donne pas, ni à voir et ni à penser clairement. On ne peut avoir à faire ici qu'aux "promesses de la liberté », comme le dit Revault d'Allonnes dans le titre de sa thèse. Il faut affirmer la vérité de la structure ou de la théorie marxiste la concernant - correspondant à l'actualité de la domination - et, en même temps, et en un certain sens, la vérité des sociologies de l'action - lorsqu'elles traitent des virtualités de la liberté ${ }^{36}$. Les deux propositions sont affirmables en même temps sans contradiction pour autant que l'on introduit entre elles la différence de l'actuel et du virtuel. La complémentarité (au sens technique) n'est dépassée que par cette affirmation simultanée de deux réalités, dont l'une est, alors qu'elle ne devrait plus être : dénonciation structurale de la domination actuelle, de ses effets idéologiques sur le discours de la liberté, de son efficacité aliénante sur les pratiques des dominés; dont l'autre n'est pas encore pleinement agissante mais qu'il faudrait faire advenir: réalité des virtualités de la liberté, qu'il faut s'employer à faire accéder à l'effectivité actuelle de leur exercice.

C'est là tout le sens d'une psychologie «compatible avec le marxisme », selon l'expression des disciples de Meyerson, Malrieu et Vernant; psychologie politique non libérale, non-elsterienne pourrait-on dire, non conforme aux schémas individualistes et anti-structuralistes tracés par Jon Elster dans sa Psychologie politique. Plus qu'à une psychologie de la liberté, on a affaire à une psychologie de la libération. Se manifestent aussi bien ici les limites du «structuralisme »: pas plus que la liberté ne réside dans l'illusion qui anime l'existence et les pensées des bourgeois distingués,

\footnotetext{
36. Très récemment, Luc Boltanski s'est efforcé d'introduire le jeu de la liberté des acteurs au sein des structures qu'analysait celui qui fut son premier maître, Pierre Bourdieu. Plus exactement, Boltanski a dans un premier temps mené un brutal «parricide » qui l'a conduit à affirmer la liberté sans les structures, en dépit des structures ou au-delà d'elles. Revenant sur son propre parcours, il réintroduit la considération des structures : le sociologue de l'action a pour ambition d'accompagner le mouvement d'affirmation des individus, qui est un mouvement critique à l'égard de certaines structures de domination (De la critique, Paris, Gallimard, 2009).
} 
elle n'est dans la simple compréhension des structures de la domination. Le sens de la liberté formelle doit être dénoncé, mais ce n'est pas pour se confier à une définition de la liberté «réelle» comme saisie de la détermination de tout un chacun par les structures du tout social. La liberté n'est pas seulement la nécessité bien comprise. Il faut accepter ce minimum de vie ou d'animation, en un mot de spontanéité, sinon active du moins réactivable, presque toujours en sommeil, mais toujours aussi éveillable sous les structures elles-mêmes - vérité du bergsonisme.

Dans la structure, il est donc deux fois question de psychologie, une première fois comme illusion, mais une deuxième fois comme vérité, ou rapport vrai à un «objet» psychologique consistant et essentiel. Dans ce dernier cas, la liberté se dit, mais non pas de ce que l'individu bourgeois, dominant, se reconnaît, ou prétend pouvoir se reconnaître et reconnaître à tout homme; plutôt de ce que les forces de travail sont susceptibles de trouver ou de retrouver, moyennant l'explosion du verrou de la domination, la disparition, éventuellement violente, de la barrière imposée par le bourgeois pour préserver son niveau et sa distinction. Ce deuxième sens suppose toute la structure du marxisme, le marxisme compris selon les canons du structuralisme. Mais, contrairement au structuralisme d'Althusser, il découvre pour la psychologie un autre sens et un autre usage que celui, idéologiquement informé et idéologiquement déformant, que proclame la bourgeoisie. La psychologie bourgeoise est cette «discipline» (en tous les sens du terme) inventée par le dominant pour masquer sa domination tout en l'exerçant. L'idéologie est alors cette illusion aux effets bien réels, qui se transmet d'individu en individu et réalise des « citoyens » tous aliénés. Mais une psychologie de la structure est toute autre. C'est une psychologie qui, forte du sens, de la vérité de la structure, et de l'illusion idéologique produite par la psychologie individualiste, en tire les conséquences pour opérer l'épanouissement des sujets en encourageant une égalisation libératrice.

Lorsque l'individualiste-libéral parle de libération, il entend : « échapper aux structures », car les structures, quelles qu'elles soient, étatiques, sociales, collectives, empêchent la seule réalité qui soit, à savoir les individus, de se manifester. Mais la «conscience» que réclament les psychologues de la libération, la « prise de conscience » qu'ils cherchent à favoriser, n'a rien de la «simple» libération à l'égard de toute communauté de structure, des institutions du collectif ou des organes du social, toutes réalités tenues - par le psychologue non-marxiste - comme contraignantes. C'est au contraire aux présupposés, typiquement individualistes, d'une telle «libération » qu'ils en ont, et à ceux qui les mettent en avant, afin de préserver leur place dans la structure, la hauteur de leur distance sociale, la supériorité de leur domination. Les psychologues de la libération entendent encourager et soulager ceux qui souffrent d'être soumis, aliénés, exploités, sans en avoir conscience, puisque tout tend, structurellement, du fait de la structure sociale, de la situation des dominants et des effets de leur idéologie, à associer conscience et liberté individuelle. Lorsque ces psychologues 
réclament une juste et enrichissante «personnalisation ${ }^{37}$ », ils ont en vue tout autre chose que l'objet de la psychologie individualiste. Ils visent ce qui, dans la structure, se présente comme classe, caste, ou groupe d'oppression, et met tout prétendant au statut de personne dans une situation délicate, fragile et précaire. Ce à quoi s'oppose la psychologie marxiste, ce n'est pas à la structure en général, mais à ce qui, et à ceux qui, dans la structure s'opposent à la personnalisation de tous.

37. Selon le processus, inverse et complémentaire de la «socialisation », profondément analysé par Ph. Malrieu, notamment dans «Personnalisation et lutte de classes », Les cahiers du Centre d'Etudes et de Recherches Marxistes, 1963, et La formation de la personnalité, avec S. Malrieu et D. Widlöcher, Paris, PUF, 1973. 